\title{
Upregulation of microRNA-206 induces apoptosis of vascular smooth muscle cells and decreases risk of atherosclerosis through modulating FOXP1
}

\author{
TAO XING ${ }^{1}$, LIXIN DU ${ }^{2}$, XIANBO ZHUANG ${ }^{2}$, LIYONG ZHANG ${ }^{1}$, JIHENG HAO ${ }^{3}$ and JIYUE WANG ${ }^{3}$ \\ Departments of ${ }^{1}$ Neurosurgery and ${ }^{2}$ Neurology, Liaocheng People's Hospital; ${ }^{3}$ Department of \\ Neurosurgery, Brain Hospital of Liaocheng, Liaocheng, Shandong 252000, P.R. China
}

Received August 20, 2015; Accepted December 9, 2016

DOI: 10.3892/etm.2017.5071

\begin{abstract}
Forkhead box protein subfamily P (FOXP) 1 has an important role in the control of gene transcription and is also reported to function as a tumor suppressor. The aim of the present study was to explore the regulatory mechanisms of atherosclerosis by investigating the function of microRNA-206 (miR-206) and the regulatory association between miR-206 and its potential target gene, FOXP1, in vascular smooth muscle cells (VSMCs). Bioinformatics tools were utilized to identify FOXP1 as a target of miR-206. Luciferase reporter analysis was used to confirm this relationship and to identify the miR-206 binding site in the FOXP1 3'-untranslated region. It was demonstrated that the relative survival rate of VSMCs was suppressed by miR-206 compared with scramble controls. Furthermore, reduced expression of miR-206 in atherosclerosis tissue samples was observed, and the mRNA and protein expression levels of FOXP1 were upregulated in atherosclerosis tissue samples, both compared with the controls, indicating a negative correlation between miR-206 and FOXP1. Additionally, when treated with miR-206 mimics, the relative survival rate of VSMCs was notably reduced, which was rescued by overexpression of FOXP1. These findings increased the understanding of the regulatory role of miR-206 in atherosclerosis in VSMCs via targeting the FOXP1 gene; therefore, intervention with miR-206 as a therapeutic technique may be a strategy for atherosclerosis treatment in the future.
\end{abstract}

\section{Introduction}

Cardiovascular disease (CVD) is one of the leading causes of mortality worldwide, and was responsible for $\sim 31.9 \%$ of

Correspondence to: Professor Xianbo Zhuang, Department of Neurology, Liaocheng People's Hospital, 45 Huashan Road, Liaocheng, Shandong 252000, P.R. China

E-mail: endarterectomyzh@163.com

Key words: microRNA-206, vascular smooth muscle cells, apoptosis, atherosclerosis, forkhead box protein subfamily $\mathrm{P}$ all mortality in 2010 (1). In the last 20 years, CVD mortality rates have decreased; however, these rates are predicted to increase again as a result of global increases in diabetes and obesity. Additionally, the prevalence of CVD has increased in developing countries, due to the acquisition of a westernized lifestyle, which is likely to contribute to the suspected increase in CVD mortality rates (2). Atherosclerosis can lead to myocardial infarction, cerebrovascular accident and peripheral vascular disease, and is now identified as an inflammatory disorder of medium and large arteries. Factors related to the development of atherosclerosis include high plasma cholesterol levels and hypertension (3).

As highly differentiated muscle cells, vascular smooth muscle cells (VSMCs) comprise the medial layer of the vessel wall, and they control blood pressure via the contraction and relaxation of vessels. VSMCs may have an important role in atherogenesis by actively participating in the control of vascular remodeling and plaque stabilization (4). Induced by various factors, the aberrant proliferation of VSMCs has been confirmed to be critical in the pathogenesis of cardiovascular diseases, such as pulmonary artery hypertension, post-angioplasty restenosis, vein graft failure and atherosclerosis $(5,6)$. Distinguishing them from many terminal differentiated cells, VSMCs have the potential of phenotypic switching, meaning VSMCs are capable of transferring between proliferative-synthetic and quiescent-contractile phenotypes, initiated by changes in the local environment $(7,8)$.

MicroRNA (miRNA) are small, endogenous, non-coding regulatory molecules of 20-22 nucleotides in length. They are able to regulate gene expression at the posttranscriptional level by binding to the 3'-untranslated region (UTR) of messenger RNA (mRNA) (8). The involvement of miRNA in the pathogenesis of various human diseases, including cancer, inflammatory diseases and cardiovascular diseases (9-13), has been widely investigated. Multiple miRNA are known to control the different phases of atherogenesis (14); they have an important role in the proliferation and differentiation of VSMCs under pathological or physiological conditions, as well as modulating both adaptive and innate immune responses within VSMCs, influencing every step of atherogenesis from plaque formation to destabilization and rupture (15). Although many miRNA have been linked to atherosclerosis, this association has only 
been repeatedly reported for some miRNA, including miR-21, -146a, -150 and -155 (16,17).

Forkhead Box P1 (FoxP1) was first identified by Shu et al (18), which was considered a glutamine rich factor that belongs to the FoxP subfamily of the fox-transcription factors. Bot et al (19) suggested that FoxP1 modulates collagen synthesis and proliferation of smooth muscle cell through downstream target of transforming growth factor (TGF)- $\beta$. FoxP1 levels may also be associated with interleukin-2, -4 and -10 expression, which are involved in plaque stability (19).

In the present study, four miRNA (miR-206, -125a, -876 and -183) that have previously been reported to be differentially expressed in atherosclerosis (20) were selected. Screening was performed to compare their ability to affect the proliferation of VSMCs with the control. It was demonstrated that miR-206 was able to substantially suppress the proliferation of VSMCs via targeting forkhead box protein subfamily P (FOXP) 1.

\section{Materials and methods}

Ethics statement. All patients involved in the present study provided their written informed consent. The present study was approved by the Ethics Committee of Liaocheng People's Hospital (Liaocheng, China).

Patient samples. A total of 22 human atherosclerotic plaques were collected from patients (age, 67.63 \pm 7.23 years; males, 15 ; females, 7) who received endarterectomy to reduce the long-term risk of stroke, and 20 normal control tissue samples were obtained from patients (age, 65.45 \pm 5.43 years; males, 12; females, 8) who received coronary artery bypass surgery in Liaocheng People's Hospital. All patients included in the present study were clinically asymptomatic. Following surgery, all tissue samples were flash-frozen in liquid nitrogen and, subsequently, stored at $-80^{\circ} \mathrm{C}$ until the experiments were conducted.

Reverse transcription-quantitative polymerase chain reaction (RT-qPCR) analysis. RNA isolated from tissue samples were subjected to an RNeasy kit (Qiagen China Co., Ltd., Shanghai, China) and an miRNeasy Mini kit (Qiagen China Co., Ltd.) to isolate miRNA and mRNA, respectively according to the manufacturer's instructions. A SYBR Green Master Mix kit and a miRCURY LNA Universal RT microRNA PCR kit (both Exiqon, Vedbæk, Denmark) for cDNA synthesis were utilized to perform RT-qPCR for miRNA and mRNA in accordance with the manufacturer's protocols. The following primers were used: miR-206, forward 5'-CCA AAGCGGAGTCTCGCAT-3' and reverse 5'-GCCTAGCAT CTTGCTTAGCTC-3'; miR-125a, forward 5'-TTGCTCCAG CAGGGTAACTG-3' and reverse 5'-GTGGTCGAGAAG CTTGTGTGA-3'; miR-876, forward 5'-ACCTGCACCCGA TTCACAG-3' and reverse 5'-TGGCAGCTCCATACTGAC CA-3'; miR-183, forward 5'-GAGAATTGTGGCGTCAAG TCA-3' and reverse 5'-CAGGTATGTTTTCCAGTGCTCC-3'; FOXP1, forward 5'-CAGCGAAACCACGAAAAGAAG-3' and reverse 5'-GGTCCACCTCTGTTAGTGATA-3'; and $\beta$-actin: Forward 5'-CAGCAAAGAAGGACACGAAAC-3' and reverse 5'-ACCGGGGATTATTCCTTCTGA-3. RT-qPCR for four miRNA (miR-206, -125a, -876 and -183) and FOXP1 was conducted using a HotStart-IT SYBR Green qPCR Master Mix with UDG (2X) (Affymetrix, Inc., Santa Clara, CA, USA) and a DNA Engine Opticon 2 Real-Time Cycler (MJ Research, Inc., Waltham, MA, USA). Subsequently, the $2^{-\Delta \Delta C q}$ method was used to quantify the expression levels of each miRNA or mRNA (18). Experiments were repeated at least three times: $\beta$-actin was the internal reference gene.

Cell culture and oligonucleotide transfection. Human aortic VSMCs (Lonza, Inc., Allendale, NJ, USA) were cultured in Dulbecco's Modified Eagle's medium (DMEM; Gibco; Thermo Fisher Scientific, Inc., Waltham, MA, USA) supplemented with $10 \%$ fetal bovine serum (FBS) (Bio-Rad Laboratories, Inc., Hercules, CA, USA). Cells were incubated at $37^{\circ} \mathrm{C}$ in an atmosphere containing $5 \% \mathrm{CO}_{2}$. Mimics for four miRNA (miR-206,-125a, -876 and -183) were purchased from Ambion (Thermo Fisher Scientific, Inc.) and transfection was performed using Lipofectamine 2000 (Invitrogen; Thermo Fisher Scientific, Inc.) according to the manufacturer's protocol.

Construction of FOXP1 plasmid. The coding sequence of FOXP1 was amplified using PCR and inserted into pcDNA3.0 (Invitrogen; Thermo Fisher Scientific, Inc.). PCR amplification was performed as follows: $94^{\circ} \mathrm{C}$ for $3 \mathrm{~min}$, followed by 30 cycles of $94^{\circ} \mathrm{C}$ for $40 \mathrm{sec}, 56^{\circ} \mathrm{C}$ for $45 \mathrm{sec}$ and $72^{\circ} \mathrm{C}$ for $60 \mathrm{sec}$, followed by terminal elongation. A DNA Engine Opticon 2 Real-Time Cycler (MJ Research, Inc.) and Taq DNA Polymerase (Invitrogen; Thermo Fisher Scientific, Inc.) were used. Insertion accuracy was confirmed via direct Sanger sequencing. The construct was subsequently transfected into VSMCs using Lipofectamine 2000.

CCK-8 assay. A cell counting kit-8 (CCK-8; Sigma-Aldrich; Merck Millipore, Darmstadt, Germany) assay was used to measure the proliferation of cells. Accordingly, $24 \mathrm{~h}$ subsequent to producing equal numbers of VSMCs, the cells were transfected with either the miR-206 mimic, or both the miR-206 mimic and the FOXP1 plasmid. Following transfection, DMEM was used to incubate the cells. Subsequently, $10 \mu \mathrm{l}$ of the CCK-8 solution was added to each well where cells were cultured for $2 \mathrm{~h}$ at the end of CCK-8 treatment. Subsequently, the absorbance value was measured at a wavelength of $450 \mathrm{~nm}$ using a microplate absorbance reader to confirm the number of viable cells.

Luciferase reporter assays. The full length of the 3'UTR of FOXP1 was amplified and cloned into an miR-reporter construct (Ambion; Thermo Fisher Scientific, Inc.). Subsequently, the potential miR-206 binding site in the 3'UTR of FOXP1 was replaced with its complementary sequence using site-directed mutagenesis (210518; Stratagene; Agilent Technologies, Inc., Santa Clara, CA, USA). For the luciferase assay, $100 \mathrm{ng} / \mathrm{ml}$ of the wild-type or mutant miR-reporter construct was co-transfected with the $100 \mathrm{nM}$ miR-206 mimics into VSMCs using Lipofectamine 2000. Following 36 h, cells were lysed and $50 \mu \mathrm{l}$ of the lysate was utilized for $\beta$-Gal assay (BioVision, Inc., Milpitas, CA, USA) and $10 \mu \mathrm{l}(2 \mathrm{X})$ was used for the luciferase assay. Assays were conducted according to the manufacturer's protocol. 
Western blot analysis. Following homogenization of the tissue samples or the cultured cells, proteins were extracted with an extraction buffer composed of radioimmunoprecipitation assay buffer, protease inhibitor cocktail and phosphatase inhibitor cocktail (Calbiochem; Merck Millipore). Total proteins were quantified by the Bradford method (21). Proteins (30 $\mu \mathrm{g})$ were separated by $10 \%$ SDS-PAGE and subsequently transferred to a nitrocellulose membrane (Merck Millipore). For blocking, $5 \%$ skimmed milk was used to block the membrane for 2 hat $37^{\circ} \mathrm{C}$ and phosphate-buffered saline was utilized to wash the membrane for $5 \mathrm{~min}$. Membranes were incubated overnight with primary antibodies against FOXP1 or $\beta$-actin $(1: 5,000$ dilution; Abcam, Cambridge, UK) or rabbit $\beta$-actin $(1: 12,000$ dilution; ab8227; Abcam) at $4^{\circ} \mathrm{C}$ overnight. Subsequently the membrane was incubated with horseradish peroxidase-linked secondary antibodies (1:15,000 dilution; ab7090; Abcam) at $37^{\circ} \mathrm{C}$ for $2 \mathrm{~h}$. Following this, the membrane was treated with enhanced chemiluminescent reagent (Amersham ECL detection system; GE Healthcare Life Sciences, Chalfont, UK). An Electrochemiluminescence Plus Western Blotting Detection System (GE Healthcare Bio-Sciences, Pittsburgh, PA, USA) was used to detect the bound antibodies and high performance chemiluminescence film (GE Healthcare Bio-Sciences) was used to detect the chemiluminiscent signals in accordance with the manufacturer's recommendation. Protein expression was normalized to $\beta$-actin and quantified by densitometry. ImageJ software (version 1.37) (National Institutes of Health; Bethesda, MD, USA) was utilized to quantify the expression level of FOXP1.

Statistical analysis. Statistical analysis was performed using SPSS 16.0 (SPSS Inc, Chicago, IL, USA). Normal distribution of data was tested using the Shapiro-Wilk test. If a normal distribution was not confirmed, the Mann-Whitney U test was used to examine differences between groups. If a normal distribution was confirmed, Student's t-test or one-way analysis of variance was used to compare the parameter between two or more groups, respectively. Categorical variables were compared using the Chi-square test. $\mathrm{P}<0.05$ was considered to indicate a statistically significant difference.

\section{Results}

miR-206 suppresses the survival of VSMCs. To explore the pathogenesis of atherosclerosis, the survival rate of VSMCs transfected with several candidate miRNA that were previously reported to be differentially expressed in atherosclerosis (including miR-206, -125a, -876 and -183) (22), was investigated. Results demonstrated that miR-206 was the only miRNA that was able to significantly suppress the growth of the VSMCs. A significantly reduced survival rate was observed in comparison with the other miRs $(\mathrm{P}<0.05$; Fig. 1$)$, indicating that miR-206 may be the one miRNA out of the four miRNA investigated that suppresses the survival of VSMCs.

miR-206 targets the conserved 3'UTR of FOXP1. Due to the evident effect of miR-206 upon VSMC survival rates, it was hypothesized that the downstream miR-206 target genes may be associated with the control of cell proliferation. Bioinformatics tools (TargetScan; http://www.targetscan.org/vert_71) were

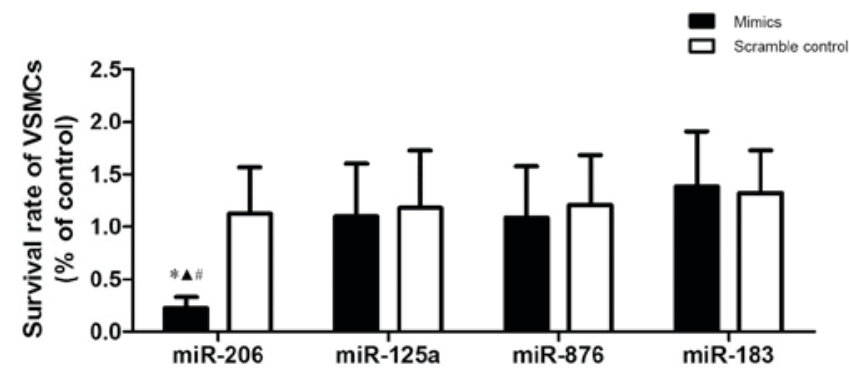

Figure 1. Relative survival rate of VSMCs transfected with four different miR-mimics. Survival rate of VSMCs was reduced by transfection with miR-206 when compared with other miRs. ${ }^{*} \mathrm{P}<0.05$ vs. miR-125a, ${ }^{\wedge} \mathrm{P}<0.05$ vs. miR-876, ${ }^{\#} \mathrm{P}<0.05$ vs. miR-183. VSMCs, vascular smooth muscle cells; miR, microRNA.

utilized to identify the potential target genes of miR-206. Two putative target sites (820-826 and 3447-3455) in the 3'UTR of the FOXP1 gene were identified as potential binding sites for miR-206 (Fig. 2), indicating that FOXP1 may be a target of miR-206.

To verify whether FOXP1 is a direct target gene of miR-206 and to determine the exact miR-206 binding site on the FOXP1 gene, two wild-type FOXP1 3'UTR segments, and two different mutants (wild-type site 820-826 and mutated site 3447-3455, defined as Mutant1 in Fig. 2; mutated site 820-826 and wild-type site 3447-3455, defined as Mutant2 in Fig. 2) of the FOXP1 3'UTR segments were cloned into different miR-luciferase reporter constructs. Following treatment with miR-206 in the luciferase reporter assay, cells co-transfected with plasmids containing the Mutant2 FOXP1 3'UTR segments exhibited similar luciferase activity when compared with the scramble controls (Fig. 3). In contrast, cells co-transfected with Mutant1 of the FOXP1 3'UTR segments demonstrated significantly reduced relative luciferase activity compared with the scramble controls, and exhibited no significant differences with cells co-transfected with wild-type FOXP1 3'UTR segments $(\mathrm{P}<0.05$; Fig. 3). This indicated that miR-206 downregulated FOXP1 expression by targeting the 820-826 putative binding site on FOXP1 3'UTR segments.

FOXP1 expression is downregulated by miR-206 in atherosclerosis tissues. To explore the mechanism by which miR-206 influences the development of atherosclerosis via targeting the predicted target FOXP1 gene, the mRNA expression level of miR-206 among the 22 atherosclerosis samples and the 20 control samples was investigated. It was demonstrated that expression of miR-206 was markedly suppressed in the atherosclerosis samples compared with control samples (Fig. 4). Additionally, RT-qPCR and western blot analysis were performed separately on atherosclerosis groups and control groups to investigate the regulatory association between miR-206 and FOXP1. This revealed significantly increased mRNA/protein expression levels of FOXP1 among atherosclerosis tissue samples compared with the normal control samples ( $\mathrm{P}<0.05$; Fig. 5A and $\mathrm{B}$, respectively), indicating the negatively correlated relationship between miR-206 and FOXP1.

FOXP1 overexpression has a potential rescue effect upon $m i R$-206. To explore the regulatory relationship between 


\begin{tabular}{|c|c|}
\hline FOXP1 3' UTR & 5' -TTCTCTAAAGTCAGAAAACATTCCG-3' \\
\hline Has-miR-206 & 3' -GGUGUGUGAAGGAAUGUAAGGU-5' \\
\hline F0XP1 Mut1 3' UTR (820-826) & 5' -TTCTCTAAAGTCAGAAATGTAAGGG-3, \\
\hline FOXP1 3' UTR & 5' -TTAATCTGTTTTTTGATACATTCCT-3' \\
\hline Has-miR-206 & 3' -GGUGLGUGAaGGAAUGUAAGGL-5' \\
\hline FOXP1 Mut2 3' UTR (3447-3455) & 5' -TTCTCTAAAGTCAGAAATGTAAGGT-3' \\
\hline
\end{tabular}

Figure 2. Two possible target sequences for microRNA-206 in the FOXP1 3'UTR and their mutants. miR, microRNA; UTR, untranslated region; Mut1, mutant 1; Mut2, mutant 2; FOXP1, forkhead box protein subfamily P.

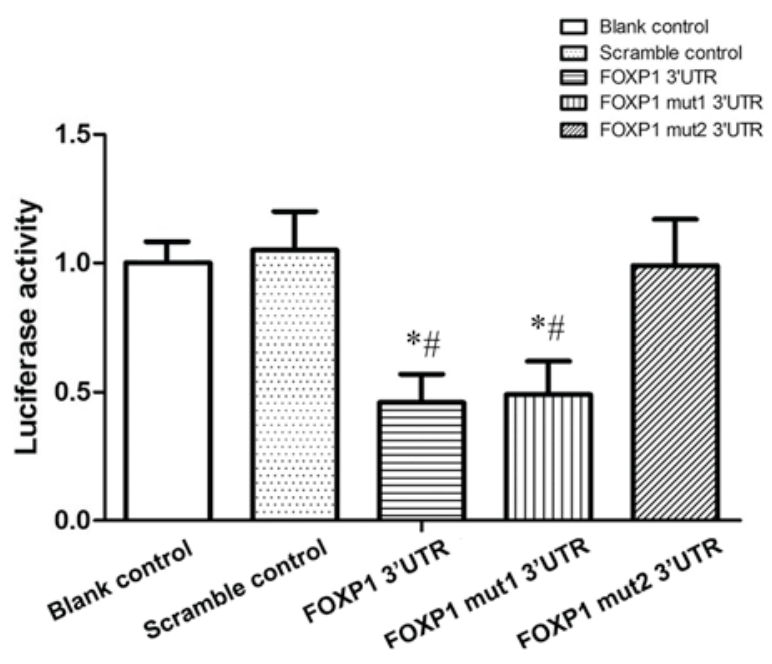

Figure 3. Luciferase activity of vascular smooth muscle cells transfected with plasmids containing the two wild-type FOXP1 3'UTR binding segments or plasmids expressing one mutant and one wild-type of each of the binding segments (mut1 and mut2). Cells transfected with plasmids containing segments of mut2 FOXP1 3'UTR/wild-type FOXP1 3'UTR exhibited no significant difference in the relative luciferase activity compared with the blank/scramble controls. In contrast, cells transfected with plasmids containing segments of wild-type FOXP1 3'UTR/Mut1 FOXP1 3'UTR demonstrated significantly decreased levels of relative luciferase activity compared with the blank/scramble controls ${ }^{*} \mathrm{P}<0.05$ vs. blank control. ${ }^{*} \mathrm{P}<0.05$ vs. scramble control. UTR, untranslated region; mut1, mutant 1 (wild-type site $820-826$ and mutated site 3447-3455); mut2, mutant 2 (mutated site 820-826 and wild-type site 3447-3455); FOXP1, forkhead box protein subfamily $\mathrm{P}$.

miR-206 and FOXP1 in vitro, the mRNA and protein expression levels of FOXP1 in VSMCs treated with miR-206 mimics were investigated. Marked decreases in protein expression (Fig. 6A) and significant decreases in mRNA expression levels of FOXP1 $(\mathrm{P}<0.05$; Fig. 6B) were detected among the miR-206 mimics treatment group compared with the scramble controls. To perform the rescue test, recombinant plasmids expressing the complete coding sequence of the FOXP1 gene were constructed and, subsequently, transfected into cells treated with miR-206 mimics. The viability of the VSMCs transfected with the scramble control, the miR-206 mimics and the miR-206 mimics with the FOXP1-expressing plasmid was determined. miR-206 transfection significantly decreased $(\mathrm{P}<0.05)$ the survival rate of the VSMCs compared with the control, while overexpression of FOXP1 almost reversed the

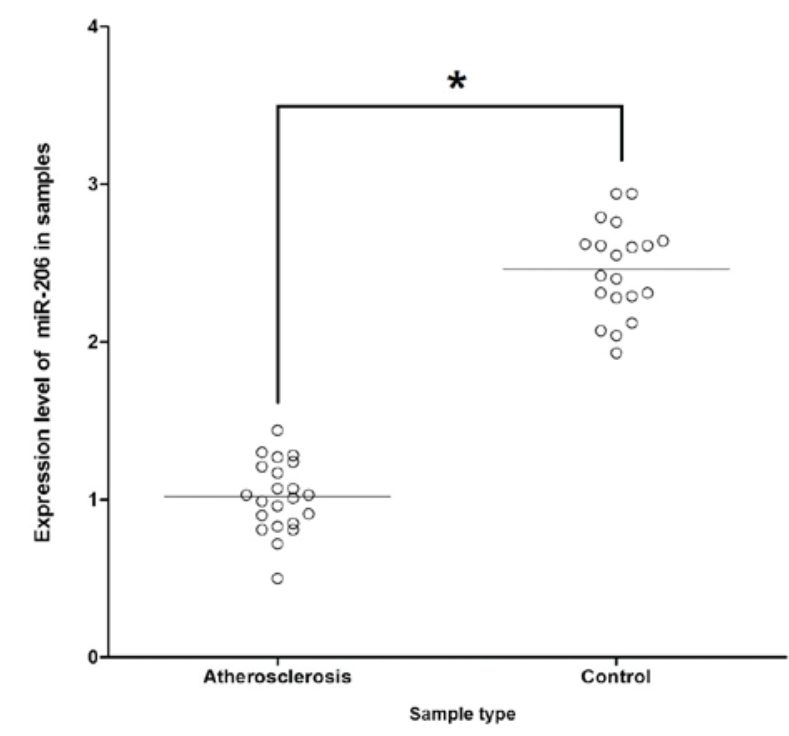

Figure 4. Expression levels of miR-206 in atherosclerosis and control samples. Expression of miR-206 was significantly reduced in atherosclerosis tissue samples compared with the controls. Horizontal line represents the standard deviation. ${ }^{*} \mathrm{P}<0.05$ vs. control. miR, microRNA.

survival decrease induced by miR-206 (Fig. 7), indicating that the miR-206 decreases VSMC viability and this is, at least partially, mediated by the target of miR-206, FOXP1.

\section{Discussion}

miR-206 is a functional miRNA in both physiological and pathological conditions. As a differentiation-related miRNA, miR-206 has been demonstrated to have a crucial role in the process of cellular differentiation. A study by Dey et al (23) demonstrated that differentiation and proliferation of myoblasts was modulated by miR-206 through its direct target, PAX7. A study by Anderson et al (24) identified miR-206 as a key regulator of osteoblast differentiation, by inhibiting the expression of the connexin-43 gene. Jalali et al (25) demonstrated that miR-206 may be a potential regulator of the differentiation and proliferation of VSMCs in pulmonary arterial hypertension. As an anti-oncogenic miRNA, miR-206 has been demonstrated to inhibit both tumorigenesis and tumor progression. A study by Song et al (26) exhibited that miR-206 is a pro-apoptotic activator of cell death that is associated with the regulation of Notch3 function and, thus, the suppression of tumor growth. In various types of cancer, miR-206 demonstrates decreased regulation $(27,28)$. By inhibiting the expression of Notch3 protein, miR-206 overexpression in HeLa cancer cells increases apoptosis (29). When compared with controls, decreased expression levels of miR-206 in mice with pulmonary hypertension induced by hypoxia has been demonstrated in experiments in vivo.

On the foundation of previous evidence, the role of miR-206 in modulating the differentiation, proliferation and apoptosis of VSMCs in vitro was investigated. In the present study, the survival rates of VSMCs transfected with several candidate microRNA, which were previously reported to be differentially expressed in atherosclerosis (including miR-206, -125a, -876 and -183$)$ (20), were investigated. miR-206 was identified 
A

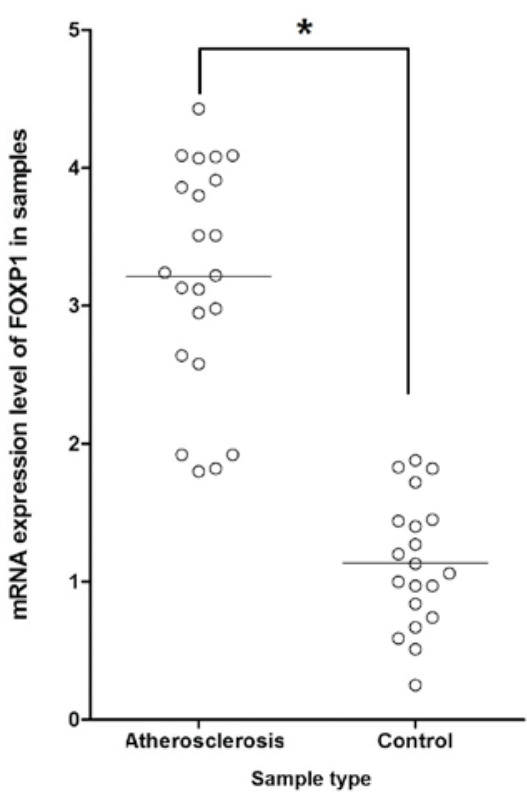

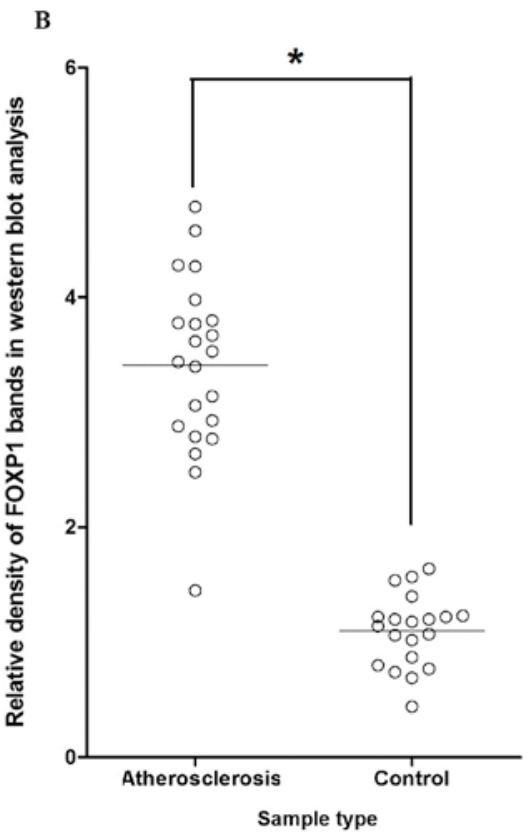

Figure 5. (A) Expression levels of FOXP1 mRNA in atherosclerosis and control samples. Expression levels of FOXP1 mRNA in VSMCs were upregulated in atherosclerosis tissue samples compared with controls. (B) Relative density of FOXP1 protein bands produced in western blot analysis from VSMCs from atherosclerosis and control samples. Horizontal lines represent the standard deviation ${ }^{*} \mathrm{P}<0.05$ vs. control. Expression levels of FOXP1 protein in VSMCs were upregulated in atherosclerosis tissue samples compared with controls. mRNA, messenger RNA; VSMCs, vascular smooth muscle cells. FOXP1, forkhead box protein subfamily $P$.

A

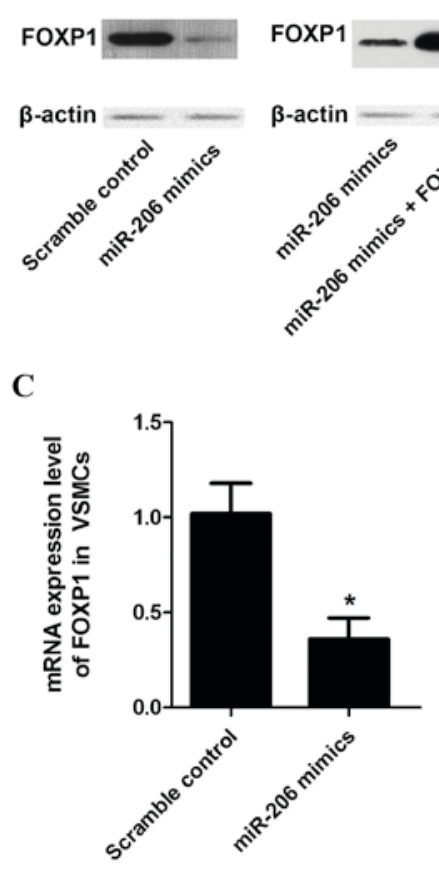

Figure 6. (A) Western blot analysis of FOXP1 protein in VSMCs transfected with miR-206. Results indicated that VSMCs transfected with miR-206 mimics presented a faint band of FOXP1 protein compared with the scramble controls. (B) Western blot analysis of VSMCs transfected with miR-206 mimics and of VSMCs transfected with miR-206 mimics along with FOXP1. Results indicated that cells co-transfected with miR-206 mimics and FOXP1 expressing recombinants exhibited a thicker/darker band compared with cells transfected with miR-206 mimics only. (C) Reverse transcription-quantitative polymerase chain reaction results of mRNA expression level of FOXP1 in VSMCs transfected with miR-206 mimics. Results indicated a significantly decreased FOXP1 mRNA expression level in cells treated with miR-206 mimics compared with the scramble controls " $\mathrm{P}<0.05$ vs. scramble control. VSMCs, vascular smooth muscle cells; miRNA, microRNA; mRNA, messenger RNA; FOXP1, forkhead box protein subfamily P.

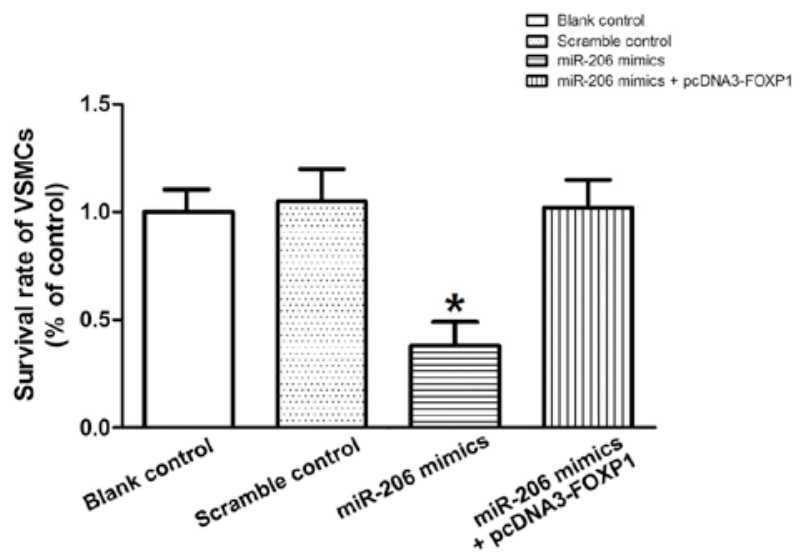

Figure 7. Survival rate of VSMCs transfected with miR-206 mimics and with miR-206 mimics plus FOXP1 expression plasmids. Relative survival rate of VSMCs transfected with miR-206 mimics was significantly reduced compared with the scramble controls, and the VSMCs co-transfected with miR-206 mimics along with FOXP1 expression plasmids exhibited no significant differences when compared with the scramble controls ${ }^{*} \mathrm{P}>0.05$ vs. scramble control. VSMCs, vascular smooth muscle cells; miR, microRNA; FOXP1, forkhead box protein subfamily P.

as the only miRNA, out of the four miRNA investigated, that could markedly suppress the growth of VSMCs. Furthermore, bioinformatics tools were utilised to identify FOXP1 as a target of miR-206 and luciferase reporter analysis was performed to confirm this relationship. Luciferase reporter analysis revealed that the binding site for miR-206 was in the 3'UTR of FOXP1. A negatively correlated relationship between miR-206 and FOXP1 was confirmed by the observation that expression of miR-206 was suppressed in the atherosclerosis samples, whereas mRNA/protein expression levels of FOXP1 were upregulated, compared with control samples. 
FOXP1, a member of the FOXP subfamily of the FOX-transcription factors, was identified in a study by Shu et al (18) as a glutamine rich transcription factor, detectable in a wide variety of fetal and adult tissue cell types. Additionally, the expression of FOXP1 has been detected in several malignant neoplasms, including B-cell lymphomas and prostate cancer (30). FOXP1 has been reported to be involved in the control of differentiation and proliferation of cardiac muscle cells, and differential effects of FOXP1 on the early and late stage of cardiac development have been demonstrated (31). Different types of cells in atherosclerotic lesions express FOXP1, which is a downstream target of TGF- $\beta$ (19). FOXP1 is predominantly expressed by smooth muscle cells (19) in atherosclerotic plaques. The role of FOXP1 in some other cell types relevant to atherosclerosis has been studied (32); however, its effect on VSMCs remains largely unknown.

In the present study, it was hypothesized that FOXP1 may be a downstream target of miR-206, and its regulatory effect on the proliferation of VSMCs may be mediated by its downstream effectors such as fibroblast growth factor and TGF- $\beta$ (33). In the present study, miR-206 transfection markedly decreased the survival rate of VSMCs compared with the controls. However, the overexpression of FOXP1 in VSMCs treated with miR-206 almost restored the survival rates, indicating that the survival-suppressing effect of miR-206 is, at least partially, mediated by its target, FOXP1.

In conclusion, the present study has provided a mechanistic model that may demonstrate the role of miR-206 and FOXP1 in the development of atherosclerotic plaques. It was demonstrated that the miR-206/FOXP1 pathway is functionally involved in the control of the proliferation of VSMCs. These results suggest that miR-206 inhibits the expression of FOXP1, resulting in decreased viability and increased apoptosis of VSMCs. Decreased levels of miR-206 release the physiologically-inhibited expression of FOXP1, resulting in the increased expression of FOXP1 and, thereby, activation of the downstream TGF- $\beta$ signalling pathway. High mRNA/protein expression levels of FOXP1 were detected in atherosclerosis samples compared with controls; therefore, miR-206 may be a protective factor in atherosclerosis by inhibiting the expression of FOXP1. FOXP1 may be a potential target for the treatment and/or prevention of acute cardiovascular events and atherosclerotic plaque formation.

\section{References}

1. Go AS, Mozaffarian D, Roger VL, Benjamin EJ, Berry JD, Blaha MJ, Dai S, Ford ES, Fox CS, Franco S, et al: Heart disease and stroke statistics-2014 update: A report from the American Heart Association. Circulation 129: e28-e292, 2014.

2. Poole-Wilson P: The prevention of cardiovascular disease worldwide: Whose task and WHO's task? Clin Med (Lond) 5: 379-384, 2005 .

3. McLaren JE, Michael DR, Ashlin TG and Ramji DP: Cytokines, macrophage lipid metabolism and foam cells: Implications for cardiovascular disease therapy. Prog Lipid Res 50: 331-347, 2011.

4. Doran AC, Meller N and McNamara CA: Role of smooth muscle cells in the initiation and early progression of atherosclerosis. Arterioscler Thromb Vasc Biol 28: 812-819, 2008.

5. Lemmens K, Doggen K and De Keulenaer GW: Role of neuregulin-1/ErbB signaling in cardiovascular physiology and disease: Implications for therapy of heart failure. Circulation 116: 954-960, 2007.
6. Marx SO, Totary-Jain H and Marks AR: Vascular smooth muscle cell proliferation in restenosis. Circ Cardiovase Interv 4: 104-111, 2011.

7. Rensen SS, Doevendans PA and van Eys GJ: Regulation and characteristics of vascular smooth muscle cell phenotypic diversity. Neth Heart J 15: 100-108, 2007.

8. Li L, Zhang HN, Chen HZ, Gao P, Zhu LH, Li HL, Lv X, Zhang QJ, Zhang R, Wang Z, et al: SIRT1 acts as a modulator of neointima formation following vascular injury in mice. Circ Res 108: 1180-1189, 2011.

9. Bartel DP: MicroRNAs: Target recognition and regulatory functions. Cell 136: 215-233, 2009.

10. Nana-Sinkam SP and Croce CM: MicroRNAs as therapeutic targets in cancer. Transl Res 157: 216-225, 2011.

11. Dai R and Ahmed SA: MicroRNA, a new paradigm for understanding immunoregulation, inflammation, and autoimmune diseases. Transl Res 157: 163-179, 2011.

12. Santovito D, Mezzetti A and Cipollone F: MicroRNAs and atherosclerosis: New actors for an old movie. Nutr Metab Cardiovasc Dis 22: 937-943, 2012.

13. Santovito D, De Nardis V, Marcantonio P, Mandolini C, Paganelli C, Vitale E, Buttitta F, Bucci M, Mezzetti A, Consoli A and Cipollone F: Plasma exosome microRNA profiling unravels a new potential modulator of adiponectin pathway in diabetes: Effect of glycemic control. J Clin Endocrinol Metab 99: E1681-E1685, 2014.

14. Nazari-Jahantigh M, Wei $\mathrm{Y}$ and Schober A: The role of microRNAs in arterial remodelling. Thromb Haemost 107: 611-618, 2012.

15. Kang $\mathrm{H}$ and Hata A: MicroRNA regulation of smooth muscle gene expression and phenotype. Curr Opin Hematol 19: 224-231, 2012.

16. Ma X, Ma C and Zheng X: MicroRNA-155 in the pathogenesis of atherosclerosis: A conflicting role? Heart Lung Circ 22: 811-818, 2013.

17. Raitoharju E, Oksala N and Lehtimäki T: MicroRNAs in the atherosclerotic plaque. Clin Chem 59: 1708-1721, 2013.

18. Shu W, Yang H, Zhang L, Lu MM and Morrisey EE: Characterization of a new subfamily of winged-helix/forkhead (Fox) genes that are expressed in the lung and act as transcriptional repressors. J Biol Chem 276: 27488-27497,2001.

19. Bot PT, Grundmann S, Goumans MJ, de Kleijn D, Moll F, de Boer O, van der Wal AC, van Soest A, de Vries JP, van Royen $\mathrm{N}$, et al: Forkhead box protein $\mathrm{P} 1$ as a downstream target of transforming growth factor- $\beta$ induces collagen synthesis and correlates with a more stable plaque phenotype. Atherosclerosis 218: 33-43, 2011.

20. Mandolini C, Santovito D, Marcantonio P, Buttitta F, Bucci M, Ucchino S, Mezzetti A and Cipollone F: Identification of microRNAs 758 and $33 \mathrm{~b}$ as potential modulators of ABCA1 expression in human atherosclerotic plaques. Nutr Metab Cardiovasc Dis 25: 202-209, 2015.

21. Ernst $\mathrm{O}$ and Zor T: Linearization of the bradford protein assay. J Vis Exp 12: pii: 1918, 2010.

22. Abeyesinghe SM, Nicol CJ, Wathes CM and Randall JM: Development of a raceway method to assess aversion of domestic fowl to concurrent stressors. Behav Processes 56: 175-194, 2001.

23. Dey BK, Gagan J and Dutta A: miR-206 and -486 induce myoblast differentiation by downregulating Pax7. Mol Cell Biol 31: 203-214, 2011.

24. Anderson C, Catoe $\mathrm{H}$ and Werner R: MIR-206 regulates connexin43 expression during skeletal muscle development. Nucleic Acids Res 34: 5863-5871, 2006.

25. Jalali S, Ramanathan GK, Parthasarathy PT, Aljubran S, Galam L, Yunus A, Garcia S, Cox RR Jr, Lockey RF and Kolliputi N: Mir-206 regulates pulmonary artery smooth muscle cell proliferation and differentiation. PLoS One 7: e46808, 2012.

26. Song G, Zhang Y and Wang L: MicroRNA-206 targets notch3, activates apoptosis, and inhibits tumor cell migration and focus formation. J Biol Chem 284: 31921-31927, 2009.

27. Chen X, Yan Q, Li S, Zhou L, Yang H, Yang Y, Liu X and Wan X: Expression of the tumor suppressor miR-206 is associated with cellular proliferative inhibition and impairs invasion in ER $\alpha$-positive endometrioid adenocarcinoma. Cancer Lett 314: 41-53, 2012.

28. Kondo N, Toyama T, Sugiura H,Fujii Y and YamashitaH: miR-206 Expression is down-regulated in estrogen receptor alpha-positive human breast cancer. Cancer Res 68: 5004-5008, 2008.

29. Chatterjee A and Hage FG: Guidelines in review: 2014 ACC/AHA guideline on perioperative cardiovascular evaluation and management of patients undergoing noncardiac surgery: A report of the American College of Cardiology/American Heart Association Task Force on practice guidelines. J Nucl Cardiol 22: 158-161, 2015. 
30. Banham AH, Beasley N, Campo E, Fernandez PL, Fidler C, Gatter K, Jones M, Mason DY, Prime JE, Trougouboff P, et al: The FOXP1 winged helix transcription factor is a novel candidate tumor suppressor gene on chromosome $3 p$. Cancer Res 61: 8820-8829, 2001.

31. Zhang Y, Li S, Yuan L, Tian Y, Weidenfeld J, Yang J, Liu F, Chokas AL and Morrisey EE: Foxp1 coordinates cardiomyocyte proliferation through both cell-autonomous and nonautonomous mechanisms. Genes Dev 24: 1746-1757, 2010.

32. Caruso P, MacLean MR, Khanin R, McClure J, Soon E, Southgate M, MacDonald RA, Greig JA, Robertson KE, Masson R, et al: Dynamic changes in lung microRNA profiles during the development of pulmonary hypertension due to chronic hypoxia and monocrotaline. Arterioscler Thromb Vasc Biol 30: 716-723, 2010.
33. Bonnet $\mathrm{S}$ and Archer SL: Potassium channel diversity in the pulmonary arteries and pulmonary veins: Implications for regulation of the pulmonary vasculature in health and during pulmonary hypertension. Pharmacol Ther 115: 56-69, 2007. 